\title{
Neues aus der Haarforschung: Einfluss von Prolaktin, Retinoiden und Transforming Growth Factor- $\beta$ auf das Haarwachstum
}

Kerstin Foitzik

New Findings in the Treatment of Alopecia: The Influence of Prolactin, Retinoids and Transforming Growth Factor- $\beta$ on Hair Growth

\section{Zusammenfassung}

Es hat sich kürzlich gezeigt, dass neben Androgenen und Östrogenen auch das Hormon Prolaktin an der Entwicklung der androgenetischen Alopezie maßgeblich beteiligt ist. Wir konnten zeigen, dass Prolaktin Haarzyklus-abhängig in der Maushaut exprimiert wird und in murinen Maushautorgankulturen in der Lage ist, vorzeitiges Katagen zu induzieren. Auch in isolierten humanen Anagen- und Katagen-Haarfollikeln wird Prolaktin und dessen Rezeptor exprimiert, und eine Behandlung mit Prolaktin über 6 Tage resultiert in ein vorzeitiges Eintreten der Haarfollikel in das Katagenstadium mit gleichzeitigem Anstieg von apoptotischen und einer verminderten Anzahl proliferierender, follikulärer Keratinozyten. Prolaktin wird - entgegen bisheriger Publikation - im Haarfollikel selbst produziert und übt lokal seine Funktionen aus. Verschiedene, einander beeinflussende Wachstumsfaktoren sind an der Regulation des Haarzyklus beteiligt. Auch TGF- $\beta$ spielt sowohl für die Haarfollikelmorphogenese als auch für den Haarzyklus eine maßgebliche Rolle. So induziert TGF- $\beta 2$ während der embryonalen Entwicklung Haarfollikelanlagen und TGF- $\beta 2$ knock-out-Mäuse zeigen eine verzögerte Haarfollikelmorphogenese mit einer verminderten Anzahl an Haarfollikeln. TGF- $\beta 1$ dagegen inhibiert die Haarfollikelmorphogenese. Während des Haarzyklus haben TGF- $\beta 1$ und TGF- $\beta 2$ hemmende Effekte auf das Haarwachstum und können Katagen in humanen Haarfollikeln induzieren. Zusätzlich hat sich gezeigt, dass Retinoid induzierter Haarausfall zumindest partiell durch TGF- $\beta 2$ vermittelt ist, der durch die Gabe von TGF- $\beta$-Antikörpern signifikant vermindert werden kann. Zusammenfassend motivieren diese Daten, sowohl Prolaktin- als auch TGF- $\beta$-Rezeptor Antagonisten als zukünftige Therapeutika in der Behandlung der androgenetischen Alopezie weiter zu untersuchen.

\section{Abstract}

It has recently been recognized, that next to androgens and estrogens also the pituitary hormone prolactin is involved in the regulation of androgenetic alopecia. We have shown that prolactin is expressed hair cycle dependent in murine skin and is capable of premature catagen induction in murine skin organ cultures. Prolactin and the prolactin receptor are expressed in isolated human hair follicles and daily treatment with prolactin for 6 days results in premature catagen induction, inhibition of hair shaft production, induction of apoptosis and decrease of proliferation in follicular keratinocytes. Prolactin is - contrary to other publications - produced in the human hair follicle and exerts its effects locally on the hair cycle. A cascade of distinct growth factors is necessary for the regulation of the hair cycle. Also TGF- $\beta$ plays an important role during hair follicle morphogenesis and cycling. During embryogenesis TGF- $\beta 2$ induces hair follicle plugs and TGF- $\beta 2$ knock-out mice show a delayed hair follicle morphogenesis along with a reduced number of hair follicles. Unlike TGF- $\beta 2$ - TGF- $\beta 1$ inhibits hair follicle morphogenesis. TGF- $\beta 1$ and 2 inhibit hair growth and are able to induce catagen in human hair follicles. In addition, retinoid induced hair loss is at least partially mediated by TGF- $\beta 2$, and can be significantly reduced by TGF- $\beta$ antibodies. In summary these data motivate to further investigate prolactin and TGF- $\beta$ receptor antagonists as possible therapeutics in the management of androgenetic alopecia. 


\section{Einleitung}

Haare spielen besonders in der heutigen Gesellschaft eine außergewöhnliche soziale Rolle. Dies wird schon allein durch die enorme Aufmerksamkeit, Energie und Kosten reflektiert, die für die Schönheit der Haare und besseres Wachstum verwendet wird. Vitalität, Jugend und Gesundheit sowie sexuelle Attraktivität wird mit kräftigem Haarwuchs verbunden. Haarstyling ist zusätzlich ein Mittel, um seine persönlichen und kulturellen Einstellungen auszudrücken. Es wird außerdem vielfach unterschätzt, dass Patienten mit Haarwachstumsstörungen in der Gesellschaft stigmatisiert werden. So ist der Leidensdruck der Patienten, die unter androgenetischer Alopezie leiden, immens und steht in keiner Relation zu dem tatsächlichen Krankheitsbild.

Die Haarforschung hat im letzten Jahrzehnt viele Faktoren entdeckt, die maßgeblich am Haarwachstum, Haarzyklus und an der Entwicklung der androgenetischen Alopezie beteiligt sind. Je mehr Erkenntnisse gewonnen wurden, desto klarer wurde die Komplexität des Miniorgans Haarfollikel. Transplantationsexperimente haben gezeigt, dass der Haarfollikel eine eigene, autonome innere chronobiologische Uhr besitzt, die man derzeit in der dermalen Papille vermutet und die den Haarzyklus auch nach Transplantation des Haarfollikels an einen anderen Ort fortführt [1]. Eine genaue Koordination verschiedener Faktoren, die sowohl Hormone als auch Wachstumsfaktoren umfassen, sind notwendig, um den rhythmischen Haarzyklus bestehend aus Anagen (Wachstumsphase), Katagen (Haarfollikelregression) und Telogen (Ruhephase) zu ermöglichen.

Die Bedeutung der Androgene und 5- $\alpha$-Reduktase in der androgenetischen Alopezie des Mannes und der Frau wurde schon in der Vergangenheit intensiv untersucht. Sie spielen zweifelsohne eine Schlüsselrolle in der Entwicklung der androgenetischen Alopezie, und es gibt auf dem Markt seit längerem 5- $\alpha$-Reduktase-Hemmer [2]. Kürzlich konnte gezeigt werden, dass Östrogene ebenfalls einen wichtigen Einfluss auf des Haarwachstum ausüben. In der Literatur wurden bisher widersprüchliche Daten in Bezug auf die Wirkung von Östrogenen auf das Haarwachstum meist in der Maus publiziert. Conrad et al. haben die Wirkung von 17- $\beta$-Östradiol auf isolierte humane Haarfollikel untersucht und herausgefunden, dass $17-\beta$-Östradiol die Anagenphase von Haarfollikeln der Okkzipitalregion bei Männern und Frauen verlängern kann, während die Haarschaftproduktion erstaunlicherweise nur bei Männern verstärkt wurde [3]. Somit hat 17- $\beta$-Östradiol - endlich auch wissenschaftlich bewiesen - einen positiven Effekt auf das Haarwachstum und findet seine Berechtigung als weit verbreitetes Therapeutikum der androgenetischen Alopezie der Frau in Form von Haarwassern.

Neben den Androgenen und Östrogenen trat in den letzten Jahren auch das Hormon Prolaktin in den Mittelpunkt des Interesses der Haarforschung. V.a. an Tieren mit saisonalabhängigem Haarzyklus wie Schaf und Ziege wurde eine wachstumsmodulierende Wirkung von Prolaktin beobachtet. Hormone entfalten ihre Wirkung durch Botenstoffe, die wiederum die Proliferation, Differenzierung oder Apoptose bestimmter Zellen regulieren. Eine Reihe an Botenstoffen wir NGF, NT-3, BMP, EGF und IGF wurden schon identifiziert, die an der Regulation des Haarzyklus betei- ligt sind [1]. Einer dieser Mediatoren ist der Wachstumsfaktor Transforming Growth factor $\beta$ (TGF- $\beta$ ) mit wichtigen Funktionen während der Haarfollikelmorphogenese und des Haarzyklus. Dieser Review focussiert sich auf die Rolle von Prolaktin und TGF- $\beta$ während des Haarzyklus und als mögliche zukünftige Therapeutika in der Behandlung des Haarausfalls.

\section{Prolaktin}

Prolaktin ist ein Hormon, das vorwiegend in der Hypophyse produziert wird und für seine mammotropen und laktotropen Funktionen während der Schwangerschaft und Stillzeit bekannt ist. Inzwischen weiß man, dass Prolaktin auch in anderen Organen des Körpers wie Lymphozyten, Plazenta, Gehirn und Mamma produziert wird und eine Vielfalt von Funktionen aufweist [4]. In der Haut bindet Prolaktin an dortige Rezeptoren, und es wurde schon seit längerem postuliert, dass Prolaktin als neuroendokriner Modulator epithelialer Proliferation und der Hautimmunabwehr fungieren könnte [5]. Beim Menschen geht eine Hyperprolaktinämie mit Hirsutismus, androgenetischer Alopezie, Amenorrhö und Infertilität einher [6]. Der Prolaktin-Rezeptor gehört zur Zytokin-Rezeptorfamilie. Vier Rezeptoren - eine lange und drei kurze Formen - sind für Prolaktin bekannt, wovon nur die lange Form in der Lage ist, das Prolaktin-Signal weiterzuleiten [7].

\section{Wirkung von Prolaktin auf den Haarzyklus}

In Schafen mit saisonal abhängigem Fellwechsel wurde beobachtet, dass Prolaktin einerseits in der Lage ist, ruhende TelogenHaarfollikel zu aktivieren (Anagen-Induktion) und andererseits, dass erhöhte Prolaktin-Plasmakonzentrationen assoziiert mit verlängerten Lichtperioden Katagen (Haarfollikelregression) induzieren können. Prolaktin scheint hier daher eine duale Rolle mit gegensätzlichen Funktionen zu spielen [8].

Mäuse besitzen wie der Mensch einen saisonalunabhängigen Haarzyklus. Transgene Mäuse, bei denen das Prolaktin-RezeptorGen ausgeschaltet wurde, zeigen Haarzyklusveränderungen und ein längeres Fell [9]. Die Haarzyklen verlaufen in diesen Mäusen beschleunigt und die Östrogenkonzentrationen im Blut sind vermindert. Es war daher bisher unklar, ob diese Haarzyklusveränderungen durch Prolaktin selbst oder indirekt durch die verminderten Östrogene bedingt sind.

Um die Rolle von Prolaktin auf das saisonalunabhängige Haarwachstum genauer aufzuklären, haben wir die Expression von Prolaktin und Prolaktin-Rezeptor-Protein und deren mRNA zunächst an der Maushaut während der einzelnen Stadien des Haarzyklus untersucht. Anschließend haben wir murine Haut, die nur Anagen-VI-Haarfollikel enthält, für zwei Tage kultiviert und mit Prolaktin behandelt, um zu sehen, ob Prolaktin einen direkten Effekt auf Haarfollikel besitzt. In einem zweiten Ansatz haben wir die Experimente auf den Menschen übertragen. Wir haben erneut die Expression von Prolaktin und Prolaktin-Rezeptor-Protein und mRNA diesmal in humanen Haarfollikeln untersucht. Anschließend wurden isolierte humane Haarfollikel über 6 Tage kultiviert und mit Prolaktin behandelt. 

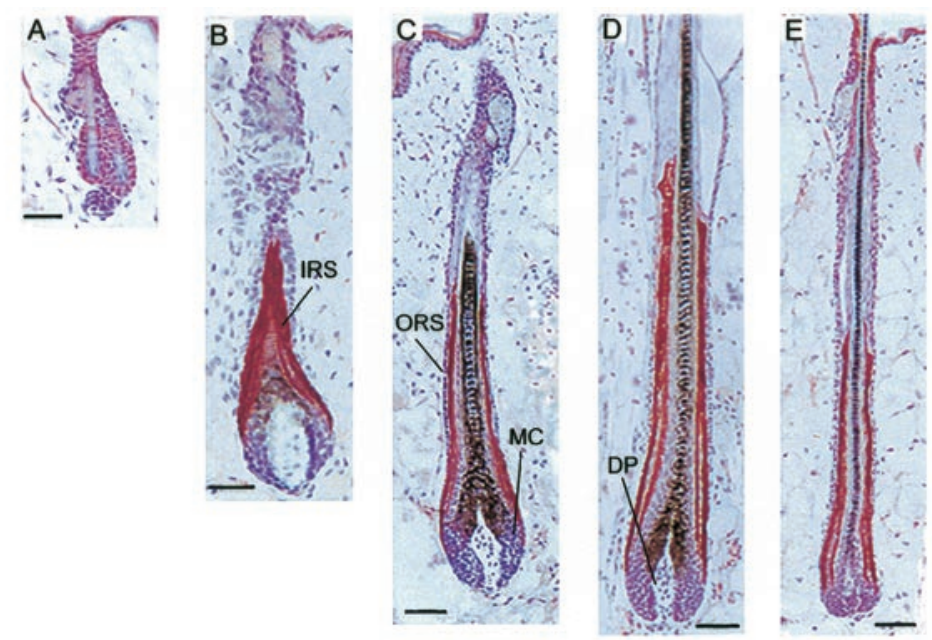

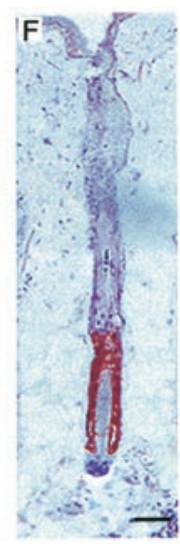

Abb. 1 Prolaktin-Expression während des depilations-induzierten murinen Haarzyklus wurde mittels ABC-Methode mit AEC+ (rot) als Substrat und Hematoxilin für die Gegenfärbung dargestellt. A: Telogen, B: Anagen III HF, C: Anagen IV, D: Anagen VI, E: Katagen III and F: Katagen VII. ORS: äußere Wurzelscheide , IRS: innere Wurzelscheide, DP: dermale Papille, and MC: Matrixzellen (aus Foitzik et al. Am. J. Pathol., 2003).

\section{Haarzyklusabhängige Expression von Prolaktin und Prolaktin-Rezeptor}

Die Prolaktin-Expression wurde immunhistologisch am depilations-induzierten Maus-Haarzyklus untersucht. Im Telogen war Prolaktin in der äußeren Wurzelscheide (ORS) nur schwach erkennbar. Im frühen Anagen wird Prolaktin in der sich entwickelnden Inneren Wurzelscheide (IRS) und im späten Anagen (IV-VI) in der IRS und der ORS exprimiert. Während des Katagens sind ORS-Keratinozyten stark positiv für Prolaktin (Abb.1). Prolaktin-Rezeptor-Expression ist ebenfalls auf epitheliale Zellen des Haarfollikels beschränkt. Die dermale Papille ist immer negativ. Während des späten Anagens und Katagens wird Prolaktin-Rezeptor in einer begrenzten Region der distalen ORS exprimiert $[9,10]$.

Mittels RT-PCR haben wir zusätzlich untersucht, ob Prolaktin im Haarfollikel selbst produziert wird und seine Wirkungen „vor Ort“ ausübt. Wir konnten zeigen, dass sowohl Prolaktin als auch Prolaktin-Rezeptor mRNA im Haarfollikel haarzyklusabhängig produziert werden.

In Maushautorgankulturen, die für $72 \mathrm{~h}$ kultiviert und mit Prolaktin behandelt wurden, konnten wir zeigen, dass Prolaktin-behandelte Haarfollikel schon deutlich weiter im Haarzyklus fortgeschritten waren und sich nach $72 \mathrm{~h}$ Kultivierung bereits vorwiegend in der Haarfollikelregression (Katagen) befanden, während die Kontrollhaarfollikel noch großteils im späten Anagen waren (Abb. 2, 3). Auch die immunhistologischen Proliferationsstudien mit Ki-67 zeigten eine signifikante Senkung der proliferierenden Matrixkeratinozyten in der Prolaktingruppe im Vergleich zur Kontrolle [10].

\section{Wirkung von Prolaktin auf humane Haarfollikel}

Die immunhistologische Analyse zeigte, dass Prolaktin-Protein auch in humanen Haarfollikeln exprimiert wird. In isolierten humanen Anagen-VI-Haarfollikeln sind ORS-Keratinozyten positiv für Prolaktin. Prolaktin-Rezeptor zeigt eine deutlich stärkere Expression in der ORS, der IRS und den Keratinozyten der Matrix in Anagen-Haarfollikeln. Im Katagen III ist die Immunreaktivität für Prolaktin intensiver als während der Wachstumsphase. Prolak-
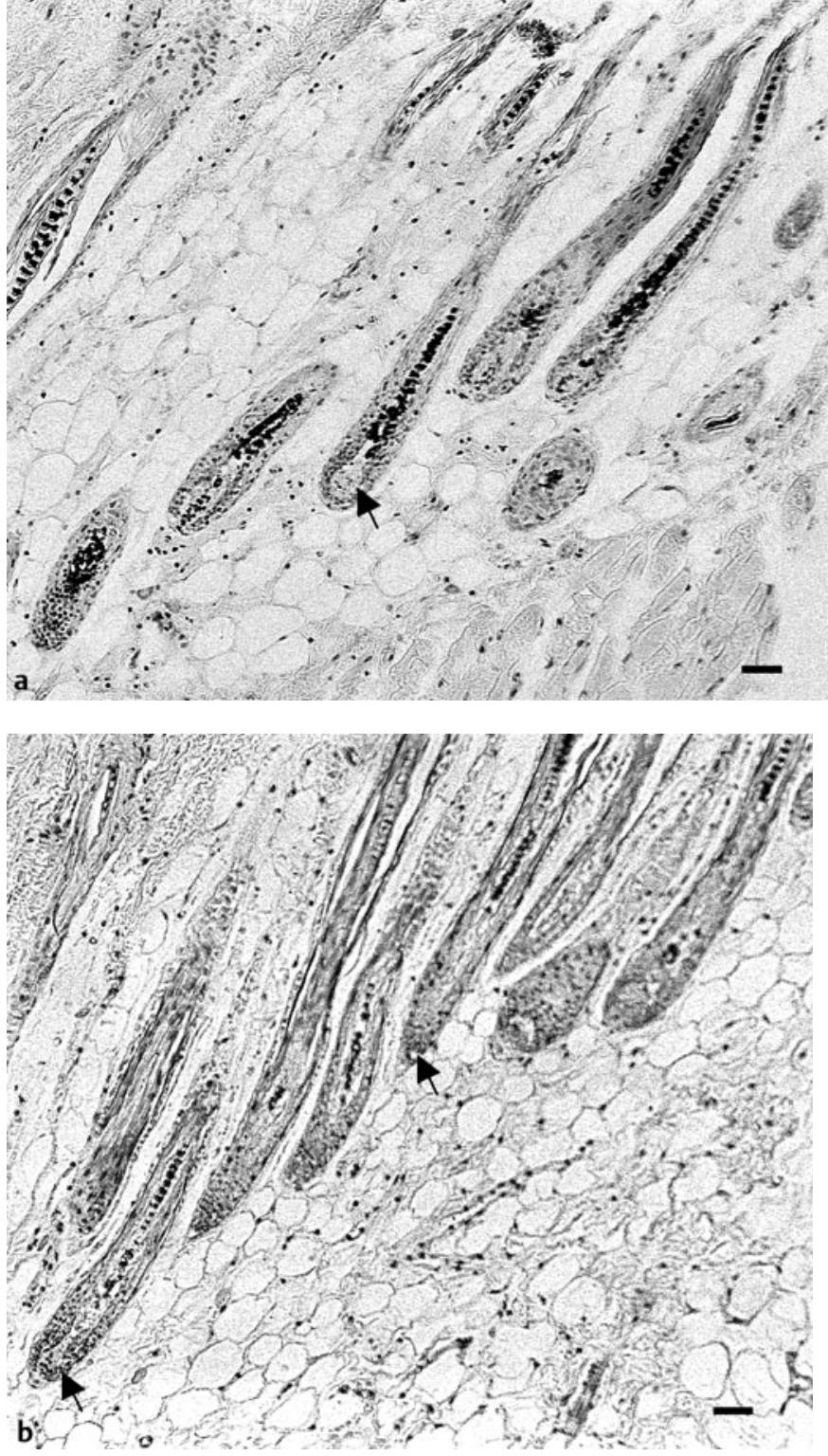

Abb. 2 H \& E Färbung für quantitative Histomorphometrie nach $72 \mathrm{~h}$ Kultivierung muriner Hautorgankultur. a Kontroll-Haarfollikel im späten Anagen VI, b Prolaktin behandelte Haarfollikel in frühem Katagen (II-III). (Pfeilspitze: Dermale Papille) (aus Foitzik et al. Am. J. Pathol., 2003). 



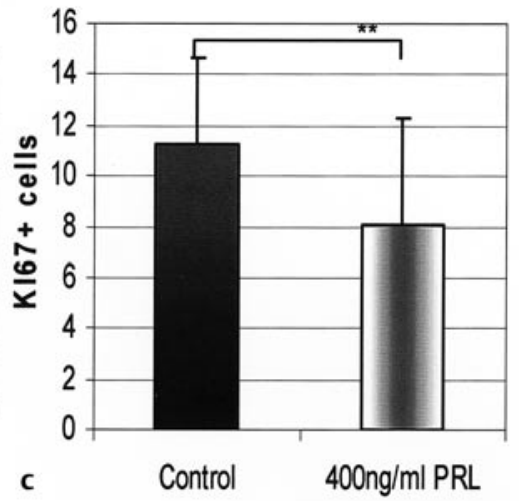

follikel von allen Gruppen wurden Haarzyklusstadien zugeordnet und jedem Stadium des Haarzyklus wurde wie folgt ein Score zugeteilt: Anagen $\mathrm{VI}=100$, frühes Katagen $=200$, mittleres Katagen $=300$. Der HCS zeigt das Mittel von allen Stadien aller Haarfollikel pro Gruppe an. P-value: ${ }^{*}<0,05$.

\section{Transforming Growth Factor- $\beta$}

ORS und der Matrix entdeckt.

Unsere oben beschriebenen Daten an der Maushaut geben Hinweise dafür, dass Prolaktin seine Wirkungen in der Haut nicht systemisch über die Hypophyse, sondern direkt am Haarfollikel ausübt. Prolaktin konnte schon in Kulturen von humanen, dermalen Fibroblasten, Keratinozyten und in Schweißdrüsen nachgewiesen werden [11]. Es wurde jedoch kürzlich berichtet, dass zwar Wachstumshormon, nicht aber Prolaktin in humaner Haut produziert wird. [12]. Wir haben mittels RT-PCR erstmals Prolaktin mRNA im humaner Haut und in isolierten Haarfollikeln nachgewiesen (Foitzik et al. in Vorber.). Die unterschiedlichen Ergebnisse könnten damit zusammenhängen, dass Slominski et al. Körperhaut mit Vellushaaren untersucht hat, während wir Skalphaut mit Terminalhaaren verwendet haben [12].

Sechs Tage wurden die humanen Haarfollikel mit Prolaktin behandelt und ausgemessen. Prolaktin-behandelte Haarfollikel wiesen eine signifikant kürzere Haarschaftverlängerung auf als die Haarfollikel der Kontrollgruppe. Während sich die Haarfollikel der Kontrollgruppe noch fast ausnahmslos im Anagen befanden, waren die Haarfollikel der Prolaktin-Gruppe schon großteils im Stadium der Haarfollikelregression. Immunhistologische Färbungen (Ki-67, TUNEL) zeigten zusätzlich, dass in der Testgruppe eine deutlich verminderte Anzahl von proliferierenden Keratinozyten in den Matrixzellen der Haarbulbi vorhanden war und dass gleichzeitig die Zahl der apoptotischen Nuklei im Haarfollikel anstieg. Prolaktin bewirkt somit nicht nur eine signifikante Inhibition der Haarschaftproduktion, sondern auch eine Induktion der Haarfollikelregression mit Hochregulation der Apoptose und Reduktion der Proliferation in follikulären Keratinozyten [31].

Diese Daten unterstützen die Hypothese, dass Prolaktin direkte lokal inhibierende Effekte auf humane Haarfollikel ausübt und dass Prolaktin-Rezeptor-Antagonisten in der Zukunft wirksame therapeutische Mittel für die Prolaktin-induzierte androgenetische Alopezie sein könnten.
Für die Mitglieder der TGF- $\beta$-Genfamilie ist eine faszinierende Vielfalt von Funktionen bekannt. Sie sind v.a. Regulatoren von Zellwachstum, Apoptose und Differenzierung und agieren als potente Morphogene während der Embryogenese [13]. Es gibt drei Isoformen für TGF- $\beta$ - TGF- $\beta 1,2$, und 3 - die eine Homologie von $80 \%$ in ihrer Aminosäuresequenz besitzen. Obwohl diverse ähnliche Eigenschaften der Isoformen in vitro bekannt sind, wurden in den TGF- $\beta$-knock-out-Mäusen nicht-überlappende Defekte beobachtet, die somit unterschiedliche Funktionen der einzelnen TGF- $\beta$-Isoformen in vivo illustrieren. Alle TGF- $\beta$-Isoformen inhibieren die Proliferation von Keratinozyten und stimulieren die Synthese und Degradation extrazellulärer Matrixproteine durch Fibroblasten. Während TGF- $\beta 1$-Apoptose in Keratinozyten induziert, ist TGF- $\beta 3$ in der Lage, Keratinozyten vor TPA induzierter Apoptose zu schützen [14].

TGF- $\beta$ Typ I- und Typ II TGF- $\beta$-Rezeptoren gehören zur Familie der Serin-Threonin-Rezeptoren. TGF- $\beta$ bindet zunächst an membranständige Typ II-Rezeptoren, die anschließend einen heteromeren Komplex mit Typ I-Rezeptoren bilden, Typ I-Rezeptoren sind dann in der Lage, das Signal über so genannte Smad Proteine an den Nukleus weiterzuleiten [15]. Daher ist die Ko-Lokalisation von TGF- $\beta$ Typ I- und Typ II-Rezeptoren eine kritische Vorraussetzung für adäquate TGF- $\beta$-Signaltransduktion.

Aufgrund ihrer wichtigen Funktionen auf die Zellregulation von Keratinozyten und extrazellulärer Matrix haben wir den Einfluss der TGF- $\beta$-Isoformen auf Haarfollikelmorphogenese und -zyklus untersucht.

\section{TGF- $\beta$ und Haarfollikelmorphogenese}

Die Haarfollikelmorphogenese bietet ein exzellentes Modell, um die Organmorphogenese generell zu studieren. Diverse Interaktionen zwischen Mesenchym und Epithel sind für die Initiation und nachfolgende Schritte der Haarfollikelentwicklung notwen- 
dig [16]. Das erste Ereignis ist durch eine epidermale Verdickung, begleitet von einer mesenchymalen Kondensation, charakterisiert. Anschließend folgt die Invagination des Epithels in die Dermis, die die papilläre Struktur umrundet. Formation einer epithelialen Plakode und anschließende mesenchymale Aggregation findet man auch in der Zahn- und Federmorphogenese, so dass man davon ausgeht, dass ähnliche Ereignisse für die Entwicklung von Haaren, Zähnen und Federn verantwortlich sind $[17,18]$. Für die Haarfollikelentwicklung sind zumindest drei induktive Signale zwischen Epithel und Mesenchym erforderlich. Heterotope Rekombinationsmodelle mit undifferenziertem Mausepithel haben gezeigt, dass das erste Signal wahrscheinlich vom Mesenchym ausgeht, an dieser Stelle ein Anhangsorgan zu bilden. Das zweite Signal hat seinen Ursprung in der Epidermis und induziert die mesenchymale Aggregation. Danach signalisiert wiederum diese mesenchymale Aggregation den umgebenden epithelialen Zellen zu differenzieren und einen reifen Haarfollikel zu bilden [18]. Die Natur dieser induktiven Signale wird derzeit aufgeklärt. Man weiß inzwischen, dass Wachstumsfaktoren wie BMP 2, 4, sonic hedgehog (shh), notch, FGF und WNT3 sowie $\beta$-Catenin an diesen ersten Schritten maßgeblich beteiligt sind [19]. Und auch für TGF- $\beta 2$ haben wir eine wichtige Rolle während der Haarfollikelmorphogenese identifiziert.

TGF- $\beta 1,2$ und 3 Isoformen sowie TGF- $\beta$-Rezeptoren zeigen ein unterschiedliches Expressionsmuster während Haarfollikelgenese und -zyklus [20-22]. Immunhistologische Expressionsstudien haben gezeigt, dass TGF- $\beta$-Typ II-Rezeptor in der Haarfollikelentwicklung schon vor den ersten morphologischen Veränderungen der Haaranlagen (Plakode-Formation) in einer Anhäufung von Keratinozyten der Epidermis exprimiert wird, die nachfolgend in die Dermis einwandern. In den nachfolgenden Stadien werden TGF- $\beta$ Typ I- und Typ II-Rezeptor von allen proliferierenden und migrierenden Keratinozyten des Haarfollikels exprimiert [20]. In der Federentwicklung wird TGF- $\beta 2$-Expression durch sonic hedgehog (shh) induziert und TGF- $\beta 2$ ist dort in der Lage, die mesenchymale Aggregation zu stimulieren [23]. Wir haben die Haarfollikelentwicklung in TGF- $\beta 1,2$ und 3 knock-outMäusen untersucht und zusätzlich in einem funktionellen Ansatz, embryonale Maushaut vor Entwicklung der Haaranlagen mit TGF- $\beta$-Protein behandelt.

Die Haut von TGF- $\beta 2$-knock-out-Mäusen zeigte eine Verzögerung der Haarfollikelmorphogenese mit Haarfollikeln in den initialen Stadien. Die Haut zeigte vorwiegend epitheliale Verdickungen und Haarplakoden, während sich die Haarfollikel in den Kontrollmäusen schon in mittleren Anagenstadien befanden. Zusätzlich zeigte sich eine Reduktion der Haarfollikel um $30 \%$ und die Dicke der Dermis von TGF- $\beta 2$-defizienten Mäusen war $50 \%$ dünner als die der Kontrolltiere [23]. TGF- $\beta 1$-defiziente Mäuse zeigten im Gegensatz dazu eine leichte Beschleunigung der Haarfollikelentwicklung und TGF- $\beta 3$-knock-out-Mäuse wiesen keinen Unterschied in der Haarfollikelmorphogenese im Vergleich zu den Kontrollen auf [23].

Um zu sehen, ob TGF- $\beta 2$ selbst in der Lage ist, Haarfollikelanlagen zu induzieren, wurde murine Embryonalhaut vor Entstehung von Haarfollikeln kultiviert und für 24 h mit TGF- $\beta 2$ behandelt. Für die embryonale Organogenese ist es entscheidend, dass ein lokaler Gradient von Wachstumsfaktoren aufgebaut wird.
TGF- $\beta 2$ wurde daher in Form von slow release beads, die TGF- $\beta 2$ hochkonzentriert und kontinuierlich über $24 \mathrm{~h}$ abgeben, auf die Hautorgankultur appliziert. Nach $24 \mathrm{~h}$ zeigte die TGF- $\beta 2$-behandelt Haut eine beschleunigte Haarfollikelentwicklung, und um die slow release beads herum konnte eine vermehrte Anzahl an initialen epithelialen Haarfollikelanlagen (hair buds) mit daneben liegender mesenchymaler Kondensation beobachtet werden (Abb. 4). Die mit Vehikel beads behandelte Haut zeigte dagegen keine initialen Haarfollikelanlagen und eine normale Epidermis [23].

TGF- $\beta 1$-überexprimierende transgene Mäuse haben dagegen eine reduzierte Anzahl an Haarfollikeln und eine verminderte Anzahl an proliferierenden Keratinozyten in der Epidermis [24], und TGF- $\beta 1$-defiziente Mäuse weisen eine vermehrte Anzahl an proliferierenden Keratinozyten auf [25]. Die stimulatorische Wirkung von TGF- $\beta 2$ ist daher eine aufregende Beobachtung, die noch weiter aufgeklärt werden muss. Eine attraktive Hypothese besteht darin, dass TGF- $\beta 2$ wahrscheinlich nicht in Keratinozyten selbst produziert wird, sondern eher in dermalen Zellen. TGF- $\beta$-Typ II-Rezeptor wird in der Haarfollikelentwicklung schon vor den ersten morphologischen Veränderungen der Haaranlagen (Plakode-Formation) in einer Anhäufung von Keratinozyten der Epidermis exprimiert, die nachfolgend in die Dermis invaginieren [20]. Wir nehmen daher an, dass TGF- $\beta 2$ an diese Rezeptoren bindet, und eine Kaskade weiterer Wachstumsfaktoren induziert, die dann für die folgenden Schritte der Haarfollikelentwicklung verantwortlich sind. Shh-defiziente Mäuse weisen einen ähnlichen Phänotyp auf wie TGF- $\beta 2$-knock-out-Mäuse mit Stillstand der Haarfollikelentwicklung im Plakode-Stadium und reduzierter Anzahl an Haarfollikeln [20]. Der stärkere Block der Haarfollikelentwicklung in shh-defizienten Mäusen und frühere Studien, die eine Induktion von TGF- $\beta 2$ durch shh gezeigt haben, schlagen vor, dass TGF- $\beta 2$ downstream von shh funktioniert. Während $\beta$-Catenin und Lef- 1 wahrscheinlich für den $\mathrm{Ab}$ stand der Haarfollikelanlagen verantwortlich sind, scheinen FGFs, notch und BMP2, 4 die Formation der Plakode und Regulation von shh zu übernehmen. Für die komplexen Vorgänge der epithelial-mesenchymalen Interaktionen während der Haarfollikelmorphogenese ist das genaue Zusammenspiel verschiedener Moleküle notwendig, und unsere Daten implizieren, dass TGF- $\beta 2$ an der Haarfollikelinduktion während der Embryogenese eine wichtige Rolle spielt. Weitere Studien sind daher notwendig herauszufinden, wie und wann TGF- $\beta 2$ mit anderen regulatorischen Wegen kommuniziert.

\section{TGF- $\beta$ und der Haarzyklus}

Katagen ist ein Prozess, der durch Apoptose (programmierter Zelltod), terminale Differenzierung, Proteolyse und Umbau der extrazellulären Matrix charakterisiert ist [25]. Auch wenn die morphologischen Kriterien des Katagens schon gut beschrieben wurden, sind die molekularbiologischen Kontrollmechanismen noch großteils unbekannt. Für verschiedene Wachstumsfaktoren wurde eine Katagen-induzierende oder Anagen-verlängernde Wirkung beschrieben. Und auch für TGF- $\beta 1$ und 2 konnte eine Funktion in der Kontrolle der Katagenregulation beobachtet werden.

Foitzik K. Neues aus der Haarforschung... Akt Dermatol 2005; 31: 109-116 

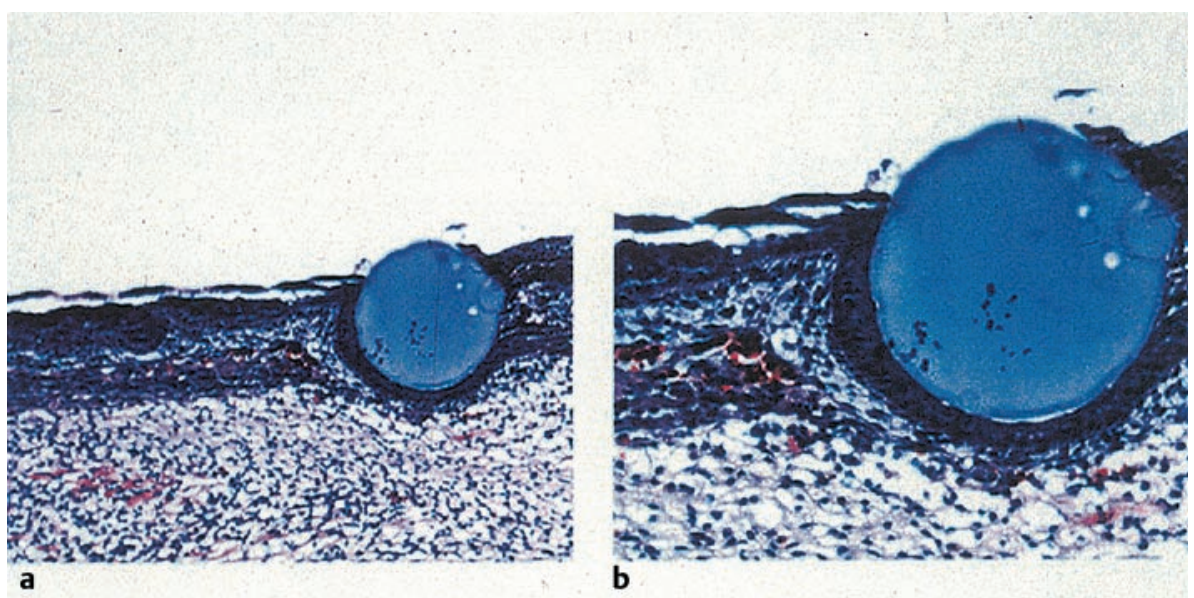

Abb. 4 a,b Embryonale Maushaut $24 \mathrm{~h}$ nach Behandlung mit Vehikel slow release bead. c, $\mathbf{d} 24 \mathrm{~h}$ nach Behandlung mit TGF- $\beta 2$ slow release bead. Um die beads sind neue Haarfollikelanlagen (Haarplakode) sichtbar. Die Epidermis ist hyperplastisch (aus Foitzik et al. Dev. Biol., 1999).
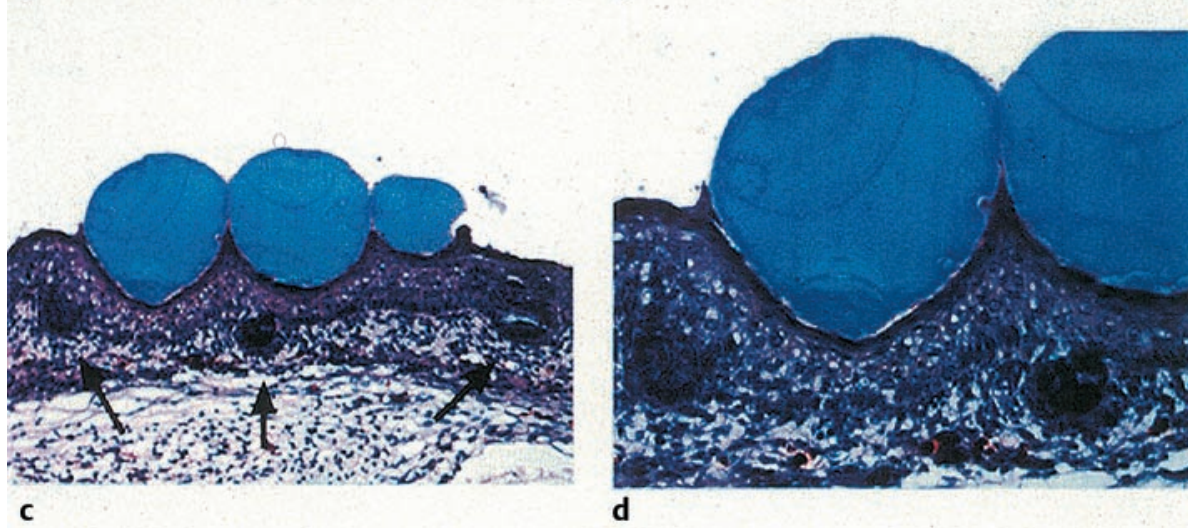

Wir haben außerdem den Haarzyklus in Mäusen, bei denen das TGF- $\beta 1-G e n$ ausgeschaltet ist, untersucht und festgestellt, dass dieser signifikant verzögert ist. Haarfollikel sind an Tag 19 nach der Geburt noch zu 50\% im deutlich verlängerten Anagen, während schon $40 \%$ der Wildtyp-Kontrollmäuse in das Telogen übergetreten sind. Damit vereinbar zeigte die Ki-67/TUNEL Immunhistochemie ebenfalls eine vermehrte Anzahl von proliferierenden und kaum apoptotische Keratinozyten in den Haarfollikeln von TGF- $\beta 1$-knock-out-Mäusen. TGF- $\beta 1$ appliziert in die Rückenhaut von C57/BL6-Mäusen mit allen Haarfollikeln im Anagen induzierte ebenfalls vorzeitiges Katagen mit Induktion von Apop-

tose follikulärer Keratinozyten [24]. Um die Zielzellen von TGF- $\beta 1$ zu entdecken und zu sehen, ob TGF- $\beta 1$ dort auch Apoptose induziert haben könnte, haben wir eine Doppelfärbung mit TGF- $\beta$-Typ II-Rezeptor und TUNEL durchgeführt und konnten eine Ko-Lokalisation von TGF- $\beta$-Typ II-Rezeptor und apoptotischen Nuklei in der ORS von Anagen Haarfollikeln und im epithelialen Strang von Katagen-Harfollikeln zeigen. Dies deutet darauf hin, dass TGF- $\beta 1$ selbst in der Lage ist, Apoptose in selektierten Keratinozyten der ORS und des epithelialen Stranges zu induzieren [25].

Im humanen isolierten Anagen-Haarfollikeln wird TGF- $\beta 1$ und 2 in der ORS der Anagen-Haarfollikel exprimiert. Im frühen Katagen wird TGF- $\beta 2$ zusätzlich in den Matrixzellen des Haarbulbus exprimiert, und im fortgeschrittenen Katagen ist TGF- $\beta 2$ erstmals auch in der dermalen Papille lokalisiert [26].

Funktionelle Daten haben zusätzlich gezeigt, dass sowohl TGF- $\beta 1$ als auch TGF- $\beta 2$ in isolierten murinen und humanen Anagen-Haarfollikeln Katagen induzieren können und die Haarschaftproduktion inhibieren [26,27]. Parallel dazu zeigen TGF$\beta 1$-knock-out-Mäuse einen deutlich beschleunigten Haarzyklus und Injektion von TGF- $\beta 1$ in vivo in murine Rückenhaut, die nur Anagan VI-Haarfollikel enthält, resultiert in einer vorzeitigen Katageninduktion [25]. Diese Ergebnisse sind insofern erstaunlich, da TGF- $\beta 2$ während der Haarfollikelmorphogenese wie oben beschrieben einen stimulierenden Effekt aufweist und auf Anagen-Haarfollikel eine gegensätzliche Katagen-induzierende Wirkung hat. Zusammenfassend kann man sagen, dass TGF- $\beta 1$ und 2 im murinen und humanen Haarfollikel vorzeitig Katagen induzieren und als wichtige Regulatoren an der Entwicklung 


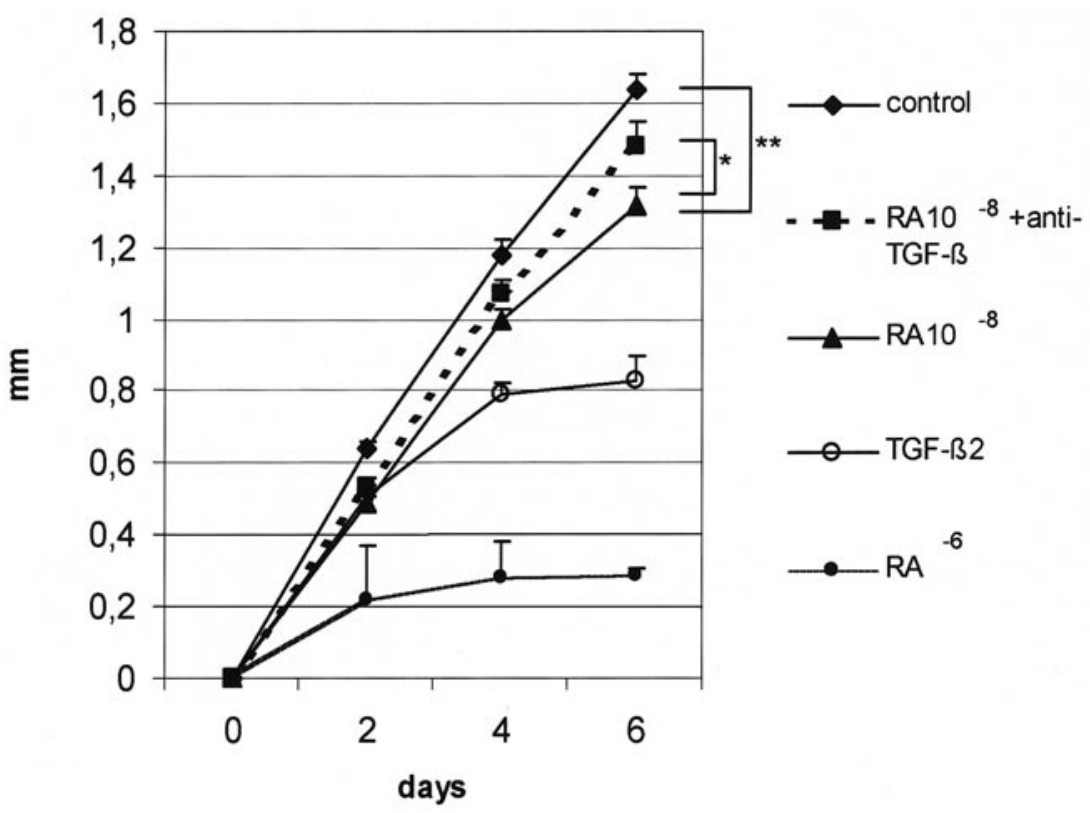

Abb. 5 a Die Graphik zeigt die Länge der Haarschäfte von Tretinoin- und TGF- $\beta 2$-behandelten Haarfollikeln im Vergleich zur Kontrollgruppe ( $\mathrm{n}=30 \mathrm{HF}$ pro Gruppe) an Tag 0 , 2, 4 and 6 in Kultur. Statistische Signifikanz wurde kalkuliert mittels Mann-WithneyU-Test: $p$-value: ${ }^{*}<0.05$, p-value: ${ }^{* *}<0,001$. b Korrespondierender Haarzyklus-Score (HCS). Kalkulation des HCS: Die Haarfollikel aller Gruppen wurden Haarzyklusstadien zugeordnet und jedem Stadium des Haarzyklus wurde wie folgt ein score zugeteilt: Anagen $\mathrm{VI}=100$, frühes Katagen $=200$, mittleres Katagen $=300$. Der HCS zeigt das Mittel von allen Stadien aller Haarfollikel pro Gruppe an. $p$-value: ${ }^{*}<0.01$ (aus Foitzik et al. J. Invest. Dermatol., in press).

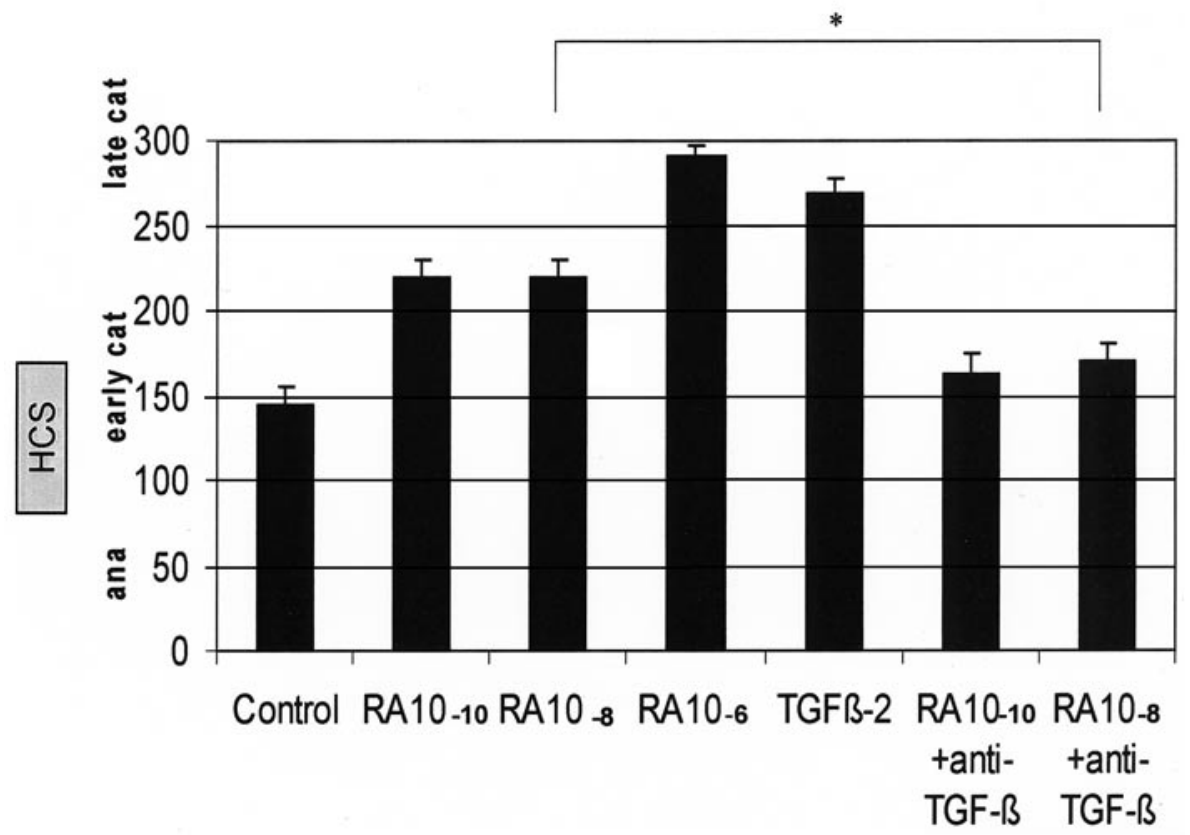

des Katagens beteiligt sind. Die Daten motivieren daher in der Zukunft zu untersuchen, ob TGF- $\beta$-Rezeptor-Antagonisten - topisch eingesetzt - als mögliche Therapeutika in der Behandlung der androgenetischen Alopezie einsetzbar sind.

\section{Retinoide und Haarwachstum}

Retinoide unterdrücken in der Haut bekanntermaßen die Sebumproduktion, Keratinisierung und Proliferation, stimulieren die Differenzierung von Keratinozyten und haben antiinflammatorische Effekte [28]. Aufgrund dieser Eigenschaften sind Retinoide ein festes Standbein in der Behandlung dermatologischer Erkrankungen wie Akne vulgaris, Ichthyosen, Psoriasis und palmoplantarer Keratosen. Diffuser Haarausfall ist eine der häufigsten und psychologisch mit am meisten beeinträchtigende $\mathrm{Ne}$ - benwirkung systemisch verabreichter Retinoide, die häufig in vorzeitiger Beendigung einer hocheffektiven systemischen Retinoid-Therapie resultiert.

Gegensätzliche Daten über die Wirkung von Retinoiden auf den Haarzyklus wurden bisher in der Literatur berichtet. So bewirkt die Einnahme von Retinoid-Tabletten diffusen Haarausfall bei Menschen, während die topische Gabe von Tretinoin in Kombination mit Minoxidil dessen Anagen-verlängernde Wirkung verbessern soll.

Um die unerwünschten Effekte von Retinoiden zu vermindern und die erwünschten Effekte zu verstärken, werden derzeit selektive Rezeptor-Subtypen-Agonisten und Antagonisten synthetisch hergestellt [29]. 
Um die bisher noch immer unklaren Effekte von Retinoiden auf den Haarfollikel genauer zu untersuchen, haben wir isolierte, humane Anagen-Skalphaut-Haarfollikel kultiviert und für 6 Tage mit Tretinoin behandelt. Nach 6 Tagen war die Haarschaftlänge der Tretinoin-behandelten Haarfollikel signifikant kürzer als die der Kontrollgruppe, und die Tretinoin behandelten Haarfollikel waren schon großteils in das Katagenstadium übergetreten. Auch die Proliferation in den Matrixzellen von Tretinoin-behandelten Anagen-Haarfollikeln war signifikant vermindert und die Apoptose hochreguliert [30]. Interessanterweise konnte der Effekt von Tretinoin auf humane Anagen-Haarfollikel signifikant durch die Zugabe von TGF- $\beta$-Antikörpern abgemildert werden, so dass sich die gleichzeitig mit Tretinoin und TGF- $\beta$-Antikörpern behandelten Haarfollikel nach 6 Tagen Kultur noch überwiegend im Anagen befanden und eine verlängerte Haarschaftproduktion zeigten (Abb. 5). Zusätzlich haben wir die Expression von TGF- $\beta 2$ als Katageninduktor und möglicher Mediator in Tretinoin-behandelten Haarfollikeln untersucht. Immunhistologisch wurde TGF- $\beta 2$-Protein in Kontrollhaarfollikeln 4 Tage nach Kultur in der ORS der Haarfollikel in der Kontrollgruppe lokalisiert. In Tretinoin-behandelten Haarfollikeln wurde eine zusätzliche Expression von TGF- $\beta 2$ in der dermalen Papille von Anagen-Haarfollikeln entdeckt [30]. Eine Untersuchung mittels Light-cycler-PCR zeigte passend dazu eine Hochregulation von TGF- $\beta 2$ mRNA im Haarbulbus im Vergleich zur Kontrollgruppe.

Diese Daten lassen darauf schließen, dass Tretinoin die Proliferation von follikulären Keratinozyten inhibiert und über Apoptose Katagen induziert. Diese Effekte von Tretinoin werden zumindest partiell durch die Hochregulation von TGF- $\beta 2$ in der dermalen Papille erzielt. Weitere Untersuchungen mit RAR $\beta$-selektiven Rezeptor-Antagonisten - die dann das Retinoid-induzierte Telogen Effluvium verhindern könnten - sind daher notwendig, um diese Hypothese zu überprüfen. Derzeit erscheint es am wahrscheinlichsten, dass topisch angewandte TGF- $\beta 2$-Antagonisten oder die lokale Blockade von TGF- $\beta$ Typ II-Rezeptoren hilfreich sein könnten, um den diffusen Haarausfall unter einer systemischen Therapie mit Retinoiden zu verhindern.

\section{Literatur}

${ }^{1}$ Stenn KS, Paus R. Controls of hair follicle cycling. Physiological Reviews 2001; 81: 449-494

${ }^{2}$ Arca E, Acikgoz G, Tastan HB, Kose O, Kurumlu Z. An open, randomized, comparative study of oral finasteride and 5\% topical minoxidil in male androgenetic alopecia. Dermatology. 2004; 209: 117-25

${ }^{3}$ Conrad F, Ohnemus U, Bodo E, Bettermann A, Paus R. Estrogens and human scalp hair growth-still more questions than answers. J Invest Dermatol. 2004; 122: 840-842

${ }^{4}$ Freeman ME, Kanyicska B, Lerant A, Nagy G. Prolactin: structure, function, and regulation of secretion. Physiological Reviews 2000; 80: $1523-1631$

5 Paus R. Does prolactin play a role in skin biology and pathology? Medical Hypotheses 1991; 36: $33-42$

${ }^{6}$ Schmidt JB. Hormonal basis of male and female androgenic alopecia: clinical relevance. Skin Pharmacology 1994; 7: 61 -66

${ }^{7}$ Kelly PA, Binart N, Freemark M et al. Prolactin receptor signal transduction pathways and actions determined in prolactin receptor knockout mice. Biochemical Society Transactions 2001; 29: 48-52

${ }^{8}$ Nixon AJ, Ford CA, Wildermoth JE et al. Regulation of prolactin receptor expression in ovine skin in relation to circulating prolactin and wool follicle growth status. J Endocrinol 2002; 172: 605-614
${ }^{9}$ Craven AJ, Ormandy CJ, Robertson FG et al. Prolactin signaling influences the timing mechanism of the hair follicle: analysis of hair growth cycles in prolactin receptor knockout mice. Endocrinology 2001; 142: $2533-2539$

${ }^{10}$ Foitzik K, Krause K, Nixon AJ et al. Prolactin and its receptor are expressed in murine hair follicle epithelium, show hair cycle-dependent expression, and induce catagen. Am J Pathol. 2003 May; 162: $1611-1612$

${ }^{11}$ Poumay Y, Jolivet G, Pittelkow MR et al. Human epidermal keratinocytes upregulate expression of the prolactin receptor after the onset of terminal differentiation, but do not respond to prolactin. Archives of Biochemistry and Biophysics 1999; 364: 247 - 253

12 Slominski A, Malarkey WB, Wortsman J, Asa SL, Carlson A. Human skin expresses growth hormone but not the prolactin gene. J Lab Clin Med 2000; 136: $476-481$

${ }^{13}$ Roberts $\mathrm{AB}$, Wakefield LM. The two faces of transforming growth factor beta in carcinogenesis. Proc Natl Acad Sci USA 2003 Jul 22; 100: 8621 - 8623. Epub 2003 Jul 14.

${ }^{14}$ Li J, Foitzik K, Calautti E, Baden H, Doetschman T, Dotto GP. TGF-beta3, but not TGF-beta1, protects keratinocytes against 12-O- tetradecanoylphorbol-13-acetate-induced cell death in vitro and in vivo. J Biol Chem 1999 Feb 12; 274: $4213-4219$

${ }^{15}$ Shi Y, Massague J. Mechanisms of TGF-beta signaling from cell membrane to the nucleus. Cell 2003 Jun 13; 113: 685-700

${ }^{16}$ Hardy MH. The secret life of the hair follicle. Trends Genet 1992; 8: $55-61$

17 Thesleff I. Epithelial-mesenchymal signalling regulating tooth morphogenesis. J Cell Sci 2003; 16: 1647-1648

18 St-Jacques B, Dassule HR, Karavanova I et al. Sonic hedgehog signaling is essential for hair development. Curr Biol 1998; 24: 1058-1068

${ }^{19}$ Botchkarev VA, Paus R. Molecular biology of hair morphogenesis: development and cycling. J Exp Zoolog Part B Mol Dev Evol 2003; 15: $164-180$

${ }^{20}$ Paus R, Foitzik K, Welker P et al. Transforming growth factor-beta receptor type I and type II expression during murine hair follicle development and cycling. J Invest Dermatol 1997; 109: 518 -526

${ }^{21}$ Welker P, Foitzik K, Bulfone-Paus S, Henz BM, Paus R. Hair cycle-dependent changes in the gene expression and protein content of transforming factor beta 1 and beta 3 in murine skin. Arch Dermatol Res 1997; 289: $554-557$

22 Pelton RW, Saxena B, Jones M, Moses HL, Gold LI. Immunohistochemical localization of TGF beta 1, TGF beta 2, and TGF beta 3 in the mouse embryo: expression patterns suggest multiple roles during embryonic development. J Cell Biol 1991; 115: 1091 - 1105

${ }^{23}$ Foitzik K, Paus R, Doetschman T, Dotto GP. The TGF-beta2 isoform is both a required and sufficient inducer of murine hair follicle morphogenesis. Dev Biol 1999; 212: 278 - 289

${ }^{24}$ Sellheyer K, Bickenbach JR, Rothnagel JA, Bundman D, Longley MA, Krieg T, Roche NS, Roberts AB. Inhibition of skin development by overexpression of transforming growth factor beta 1 in the epidermis of transgenic mice. Proc Natl Acad Sci USA 1993; 1: 5237-5241

${ }^{25}$ Foitzik K, Lindner G, Mueller-Roever S et al. Control of murine hair follicle regression (catagen) by TGF-beta1 in vivo. FASEB Journal 2000; 14: $752-760$

${ }^{26}$ Soma T, Tsuji Y, Hibino T. Involvement of transforming growth factorbeta2 in catagen induction during the human hair cycle. J Invest Dermatol 2002; 118: 993 - 997

${ }^{27}$ Philpott MP, Sanders D, Westgate GE, Kealey T. Human hair growth in vitro: a model for the study of hair follicle biology. J Dermatol Sci 1994; 7 Suppl: S55 - 72

${ }^{28}$ Marill J, Idres N, Capron CC et al. Retinoic Acid metabolism and mechanism of action: a review. Curr Drug Metab 2003; 4: 1-10

${ }^{29}$ Klaholz BP, Mitschler A, Moras D. Structural basis for isotype selectivity of the human retinoic acid nuclear receptor. J Mol Biol. 2000; 302: $155-170$

${ }^{30}$ Foitzik K, Spexard T, Nakamura M et al. Towards dissecting the pathogenesis of retinoid-induced hair loss: all-trans retinoic acid induces premature hair follicle regression (catagen) by upregulation of TGF- $\beta 2$ in the dermal papilla. J Invest Dermatol 2005, in press

${ }^{31}$ Krause K, Paus R, Nakamura M, Foitzik K. Expression of prolactin mRNA and protein in human skin and its role in catagen control of human hair fallicles. Arch Dermatol Res 2002; 294:65: P201 